\title{
PREVALÊNCIA DE MALFORMAÇÕES CONGÊNITAS E FATORES DE RISCO NA CIDADE DE CAJAZEIRAS - PARAÍBA, NO PERÍODO DE 2010 A 2016
}

\author{
PREVALENCE OF CONGENITAL MALFORMATIONS AND RISK \\ FACTORS IN THE CITY OF CAJAZEIRAS - PARAÍBA FROM 2010 TO \\ 2016
}

\author{
Wigna Luana de Figueirêdo Pimenta ${ }^{1}$ \\ Vanessa Erika Ferreira Abrantes Coutinho ${ }^{2}$ \\ Bruna Vieira Lins Campos ${ }^{3}$ \\ Otávio Soares Nascimento ${ }^{4}$ \\ Mariana Gomes Pinto 5 \\ Anne Carolinne da Silva Linhares ${ }^{6}$
}

RESUMO: Objetivo: Verificar a prevalência de malformações congênitas e possíveis fatores de risco materno na cidade de Cajazeiras-Paraíba, no período de 2010 a 2016. Método: Foi realizado o preenchimento de um formulário através de coleta de dados das Declarações de nascidos vivos, onde foi delineado a prevalência existente de malformações congênitas na cidade Cajazeiras e sua associação a fatores de risco. Foram incluídos no estudo todos os registros de nascidos vivos com má formação ocorrido na Maternidade Dr. Deodato Cartaxo localizada na cidade de Cajazeiras-PB, entre o período de 01 de janeiro de 2010 a 31 de agosto de 2016 e excluídos todos os nascimentos ocorridos em outras maternidades, cujos bebês após seu nascimento, foram atendidos por alguma razão na maternidade em estudo. Resultados e discussão: Foram constatados 35 casos de malformações congênitas entre os anos de 2010 e 2016 na cidade de Cajazeiras$\mathrm{PB}$, o que representa uma porcentagem de $0,27 \%$ do total de bebês que nasceram nesse período. As malformações mais encontradas foram as do sistema

\footnotetext{
1 Biomédica. Pós-Graduanda Especialização em Hematologia Clínica no Centro Universitário Leão Sampaio. Discente do Curso de Farmácia na Faculdade São Francisco da Paraíba.

${ }^{2}$ Bacharel e Licenciada em Ciências Biológicas. Mestre em Biologia Celular e Molecular. Docente da Faculdade Santa Maria - FSM.

${ }^{3}$ Biomédica. Pós-Graduanda Especialização em Hematologia Clínica no Centro Universitário Leão Sampaio.

${ }^{4}$ Biomédico graduado pela Faculdade Santa Maria - Cajazeiras, PB.

${ }^{5}$ Acadêmica do curso de Fisioterapia na Faculdade Santa Maria - Cajazeiras, PB.

${ }^{6}$ Biomédica. Pós Graduanda em Microbiologia Clínica na Faculdade Integrada de Patos - FIP.
} 
osteomuscular $(31,6 \%)$ com predominância da deformidade do pé. Os dados maternos apontaram que $60 \%$ das mulheres foram mães com idades entre 19 e 35 anos, $71,4 \%$ eram solteiras, $37,1 \%$ eram agricultoras e $60 \%$ fizeram mais de sete consultas pré-natais. Os partos cesáreos foram a maioria com 54,3\%. Não se observou relação estatísticas entre algumas variáveis materna em relação as malformações o que pode remeter que as MC detectadas neste estudo possam ser inferentes de fatores genéticos. A quantidade significativa de Declarações de Nascido Vivo analisadas que não continham o campo de presença de malformação preenchido, assim como as DNV que continham mais que não possuíam a descrição do tipo de malformação congênita deve ser ressaltado. Conclusão: A baixa prevalência de malformações congênitas detectadas neste estudo pode estar associada a subnotificação dos casos nas Declarações de Nascidos vivos. Há uma necessidade de melhoria no preenchimento da DNV, o que pode ocorrer através de capacitação dos profissionais envolvidos no seu preenchimento, assim como profissionais de saúde treinados corretamente para atuarem na identificação e notificação das malformações congênitas.

Palavras chave: Malformação congênita. Recém-nascido. Prevalência. Fatores de risco.

ABSTRACT: Objective: To verify the prevalence of congenital malformations and possible maternal risk factors in the city of Cajazeiras-Paraiba, from 2010 to 2016. Method: A form was completed by collecting data from Declarations of live births, where it was The prevalence of congenital malformations in the city of Cajazeiras and its association with risk factors was outlined. We included in the study all records of live births with malformation occurred at Dr. Deodato Cartaxo Maternity located in the city of Cajazeiras-PB, from January 1, 2010 to August 31, 2016 and excluded all births occurred in other maternity hospitals, whose babies after birth, were attended for some reason at the maternity hospital under study. Results and discussion: We found 35 cases of congenital malformations between 2010 and 2016 in the city of Cajazeiras- $P B$, which represents a percentage of $0.27 \%$ of the total babies born in this period. The most common malformations were those of the musculoskeletal system (31.6\%) with a predominance of foot deformity. Maternal data indicated that $60 \%$ of women were mothers aged 19 to 35 years, $71.4 \%$ were single, $37.1 \%$ were farmers and $60 \%$ had more than seven antenatal consultations. Cesarean deliveries were the majority with 54.3\%. No statistical relationship was observed between some maternal variables in relation to malformations, which may indicate that the $M C$ detected in this study may be inferred from genetic factors. The significant amount of Live Birth Statements analyzed that did not contain the filled malformation presence field, as well as DNVs that contained more than did not have the description of the type of congenital malformation should be highlighted. Conclusion: The low prevalence of congenital malformations detected in this study may be associated with underreporting of cases in Live Birth Statements. There is a need for improvement in the completion of DNV, which may occur through training of professionals involved in its completion, as well as health professionals properly trained to act in the identification and notification of congenital malformations.

Keywords: Congenital malformation. Newborn. Prevalence. Risk factors. 


\section{INTRODUÇÃO}

Malformação congênita $(\mathrm{MC})$ pode ser definida como qualquer defeito presente na estrutura anatômica regular ao nascer. Engloba toda anomalia funcional ou estrutural da evolução do feto, causado por fatores pré-natais. Pode ocasionar inaptidão física ou mental no recém-nascido e no que diz respeito às suas consequentes manifestações, ir de quadros brandos até quadros gravíssimos, com potencial risco de óbito da criança acometida (NUNES; TAVARES, 2010).

Podem ser detectadas ainda no estágio gestacional, na fase neonatal, ou ainda em tempo considerável posterior ao nascimento da criança, acarretando danos permanentes e irreversíveis de desenvolvimento ou de receptividade social (BRITO et al., 2010).

No Brasil, entre os principais motivos de mortalidade infantil, estão as malformações congênitas, ocupando o segundo lugar como fator causador de óbitos de crianças com menos de um ano de vida, demonstrando necessidade de estratégias peculiares na política de saúde (PIMENTA; CALIL; KREBS, 2010). Além da alta taxa de mortalidade, recém-nascidos que possuem algum tipo de malformação congênita, apresentam maior morbidade, que por sua vez, pode ser descrita como risco para desenvolvimento de complicações clínicas referentes à quantidade de internações e à gravidade das intercorrências (RAMOS; OLIVEIRA; CARDOSO, 2008).

As MC podem ser classificadas como isoladas ou associadas, simples ou múltiplas (FONTOURA; CARDOSO, 2014), e também como maiores, que diz respeito aos defeitos mais graves, letais ou que causam danos significativos à função ou à imagem, o que acarreta a necessidade de tratamento médico ou cirúrgico, podendo chegar a ser letal (ARRUDA; AMORIM; SOUZA, 2008).

Um grande número de casos possui causa desconhecida, porém cerca de $40 \%$ das malformações podem ser relacionadas às causas genéticas (anomalias cromossômicas), ambientais (teratógenos) e multifatoriais ou mistas (genética e 
ambiental) (FONTOURA; CARDOSO, 2014), e possuir fatores relacionados como relata a literatura que podem ser descritos da seguinte ordem: condições socioeconômicas, deficiências nutricionais, traumatismos, idade da mãe, entre outros (REIS; ALVES, 2014).

Em busca de conseguir informações das MC em estudo populacional e para que estes conhecimentos não se limitassem apenas aos dados relacionados aos hospitais, como por exemplo a Rede de Estudo Colaborativo Latino-Americano de Malformações Congênitas (ECLAM), criada em 1967 e que refere-se a um programa de investigação clínica e epidemiológica das anomalias congênitas e suas causas em nascimentos nos hospitais latino-americanos (NUNES; TAVARES, 2010), o Ministério da Saúde estabeleceu, no ano de 2000, a criação do campo 34 na Declaração de Nascidos Vivos (DNV), que pertence ao Sistema de Informações sobre Nascidos Vivos (SINASC), de disposição nacional. Neste campo deve constar o registro de ausência ou existência de "malformação congênita e / ou anomalia cromossômica" (NHONCANSE; MELO, 2012).

Apesar desse sistema de informação já utilizado, ainda há carência de conhecimento sobre as MC no Brasil (REIS; FERRARI, 2014), principalmente informações à população sobre fatores de riscos que podem acarretar tal problema e sobre as principais medidas preventivas. Dessa forma é de extremo valor o envolvimento de pesquisadores e profissionais da saúde em temas que compreendem as malformações congênitas, buscando os fatores determinantes e predisponentes dessas anomalias, assim como o envolvimento dos mesmos com autoridades em saúde, para a redução dos casos de nascimentos de crianças com malformações, bem como, o número de óbitos infantis e perinatais, com orientações das mães e população, de modo a reduzir esta problemática na saúde pública (FONTOURA; CARDOSO, 2014).

A carência de dados descritos na literatura sobre a prevalência de malformações congênitas na cidade de Cajazeiras-PB e seus possíveis fatores de riscos, foram questões norteadoras para este estudo, que possui como objetivo verificar a prevalência de malformações congênitas e possíveis fatores de risco materno na cidade de Cajazeiras-Paraíba, no período de 2010 a 2016, para que a partir destas informações, sejam identificados grupos de risco, diagnósticos 
precoces, tratamento adequado e principalmente prevenção de casos através de planejamentos das ações de saúde em todos os níveis de atenção, assim como a diminuição da letalidade.

\section{MATERIAIS E MÉTODOS}

Trata-se de um estudo descritivo, transversal e documental, com abordagem quantitativa, realizado na cidade de Cajazeiras, Estado da Paraíba, através de coleta de dados presentes nas Declarações de nascidos vivos - DNV, que trata-se de um formulário de preenchimento obrigatório institucional de origem pública ou privada, para cada criança nascida viva e através de dados contidos no Departamento de Informática do Sistema Único de Saúde (DATASUS), fornecidos pelo Departamento de Vigilância Epidemiológica da Secretária Municipal de Saúde de Cajazeiras.

A população estudada foi composta por todos os bebês nascidos na maternidade Dr. Deodato Cartaxo de Cajazeiras - Paraíba no período entre 01 de janeiro de 2010 a 31 de agosto de 2016.

Os dados coletados das declarações de nascidos vivos foram transcritos para um formulário contendo variáveis dos recém-nascidos como ano do nascimento, sexo, raça, índice de Apgar no quinto minuto distribuído em $\leq 7$ ou $>7$, peso ao nascer divididos em baixo peso $(<2,500 \mathrm{~kg})$, peso normal $(\geq 2,500 \mathrm{~kg}$ até 4,000 kg) e sobrepeso (> 4,000 kg), presença de malformação congênita ou cromossômica. As variáveis relacionadas a mãe foram idade, dividida em três categorias mães adolescentes (<19 anos), mães jovens (entre 19 e 35 anos) e mães tardias (>35 anos), estado civil dividido em mãe solteira (viúva ou separada) e casada (união consensual), ocupação atual, tipo de parto e número de consultas pré-natal separados em nenhuma consulta, menos de seis consultas e mais de 7 consultas.

Foram incluídos no estudo todos os registros de nascidos vivos com má formação ocorrido na Maternidade Dr. Deodato Cartaxo localizada na cidade de Cajazeiras-PB, entre o período de 01 de janeiro de 2010 a 31 de agosto de 2016 e excluídos todos os nascimentos ocorridos em outras maternidades, cujos bebês 
após seu nascimento, foram atendidos por alguma razão na maternidade em estudo. Utilizou-se a Classificação Internacional de Doenças e Problemas Relacionados à Saúde, décima revisão (CID-10), capítulo XVII, para identificar as malformações congênitas encontradas e separa-las em categorias, uma vez que são vários diagnósticos (OMS, 2008).

As variáveis maternas e as malformações congênitas foram testadas com o Qui-quadrado usando o programa SPSS. Os dados coletados foram organizados e sintetizados através de tabulação feita no Microsoft Office Excel versão 2010, transformando dados brutos em dados mais simples com auxílio de gráficos e planilhas eletrônicas para que houvesse uma melhor compreensão e conclusão das informações coletadas.

Esta pesquisa foi submetida à apreciação pelo Comitê de Ética em pesquisa (CEP) da Faculdade Santa Maria da cidade de Cajazeiras-PB e aprovada mediante parecer de número 1.763.244, conforme é estabelecido na Resolução 466/12 do Conselho Nacional de Saúde. Os dados foram coletados das Declarações de Nascidos Vivos mediante autorização dada pelo Secretário de Saúde através da assinatura dos termos de anuência e corresponsabilidade, bem como o termo de permissão para uso de dados.

\section{RESULTADOS E DISCUSSÃO}

Durante o período de 01 de janeiro de 2010 a 31 de agosto de 2016 foram registradas 12.850 DNV referentes a nascimentos na Maternidade Dr. Deodato Cartaxo localizada na cidade de Cajazeiras. Desse total, 12.667 estavam preenchidas corretamente e 183 não apresentavam preenchimento em relação ao campo de malformações congênitas. Nas 12.667 foram encontradas durante a análise das Declarações de Nascidos Vivos, 35 casos de Malformações congênitas o que corresponde a $0,27 \%$ de casos de Malformações nesse período. Observou-se ainda que durante o período estudado apesar de ocorrer oscilações no número de 
MC, houve uma diminuição ao longo dos anos, onde podemos observar melhor estes dados no gráfico 1.

Durante o período de 2010 a 2016, os maiores números de malformações ocorreram nos anos de 2011 e 2013, que apresentaram 9 (25,7\%) casos de malformações em cada ano. A proporção de recém-nascidos com alguma malformação no município de Cajazeiras é divergente da encontrada em outros estudos que apontaram proporção de 0,4 a $0,8 \%$ dos nascidos vivos (MELO et al., 2010).

Gráfico 1 - Prevalência de malformação congênita na cidade de Cajazeiras - PB.

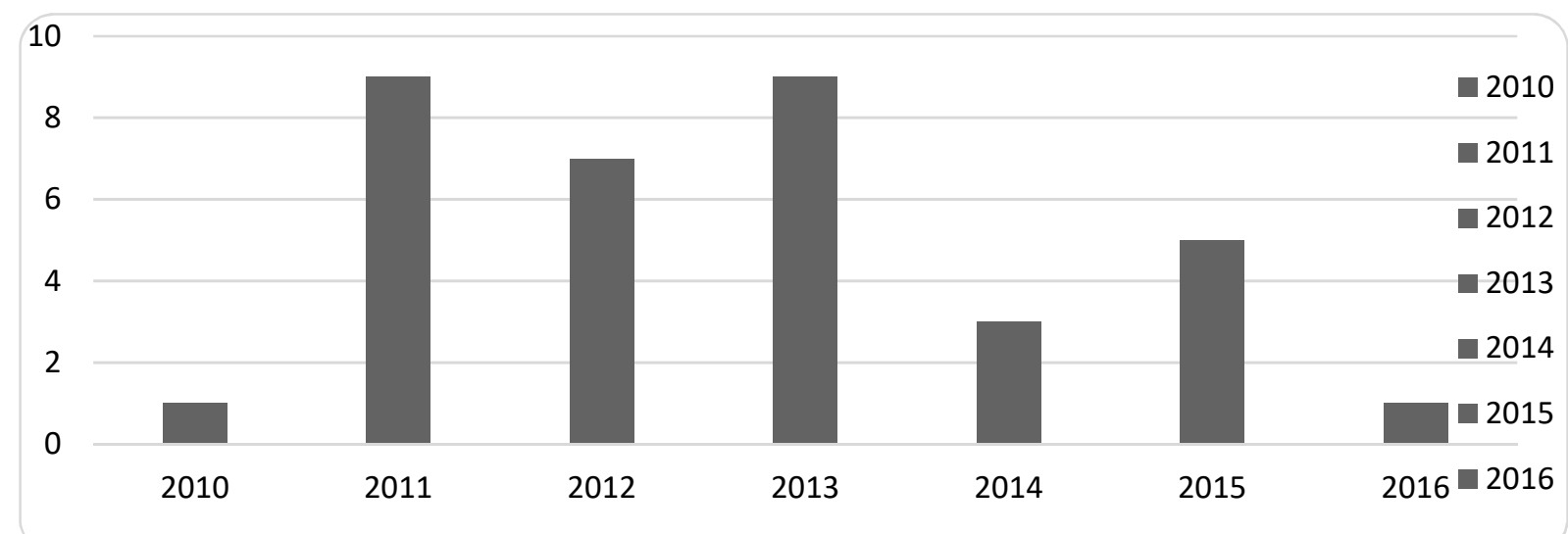

Fonte: PIMENTAL et al., 2019.

A baixa proporção de casos de Malformações congênitas na cidade de Cajazeiras durante os anos estudados pode ser devido à subnotificação, uma vez que $1,4 \%$ das $\mathrm{DN}$ analisadas apresentavam o campo destinado à notificação de Malformação em branco. 
Tabela 1 - Distribuição das malformações congênitas detectadas na cidade de Cajazeiras, PB, Brasil, entre os anos de 2010 a 2016.

\begin{tabular}{clcc}
\hline CID & \multicolumn{1}{c}{ Tipos de Malformações Congênitas } & N & $\%$ \\
\hline Q00 & Anencefalia & 1 & 2,9 \\
Q02 & Microcefalia & 1 & 2,9 \\
Q16.9 & Malf. Cong. do ouvido NE causando comprometimento do & 1 & 2,9 \\
Q17.3 & Ouvido & 1 & 2,9 \\
Q18 & Seio, fístula e cisto de origem branquial & 1 & 2,9 \\
Q35 & Fenda palatina não especificada & 1 & 2,9 \\
Q37 & Fenda do palato duro com fenda labial & 4 & 11,4 \\
Q42.3 & Ausência, atresia e estenosa cong. do ânus, sem fístula & 1 & 2,9 \\
Q53 & Testículo Ectópico & 1 & 2,9 \\
Q54.8 & Outras hipospádia & 1 & 2,9 \\
Q54.9 & Hipospádia não especificada & 1 & 2,9 \\
Q55.5 & Ausência e aplasia congênita do pênis & 1 & 2,9 \\
Q55.8 & Outra Malf. Cong. especifica dos órgãos genitais & 1 & 2,9 \\
Q66 & Pé torto eqüinovaro & 5 & 14,3 \\
Q66.1 & Pé torto calcaneovaro & 1 & 2,9 \\
Q66.8 & Outra deformidade congênita do pé & 1 & 2,9 \\
Q66.9 & Deformidade congênita NE do pé & 2 & 5,7 \\
Q67 & Assimetria Facial & 1 & 2,9 \\
Q79.3 & Gastrosquise & 1 & 2,9 \\
Q87.8 & Outras síndromes com malformações congênitas NCOP & 1 & 2,9 \\
Q98.9 & Anomalia NE dos cromossomos sexuais, fenótipo & 1 & 2,9 \\
& masculino & 6 & 16,4 \\
\hline & Malformações não especificadas & 35 & 100 \\
\hline & Total & & \\
\hline
\end{tabular}

Fonte: PIMENTA et al., 2019.

As malformações mais encontradas foram as do sistema osteomuscular (31,6\%) com predominância da deformidade do pé. Segundo Reis (2010), a predominância de malformações deste tipo de sistemas pode estar relacionada com a facilidade de diagnóstico, assim como o diagnóstico precoce, pois se tratam de malformações macrossômicas visíveis e facilmente detectáveis ao exame físico.

Quanto aos dados maternos o estudo apontou que 21 (60\%) mulheres foram mães com a idade entre 19 e 35 anos, faixa etária considerada fora de risco, seguidas de $9(25,7 \%)$ que foram mães com menos de 19 anos e $5(14,3 \%)$ com mais de 35 anos. Este resultado corrobora com o encontrado no estudo de 
Rodrigues et.al, 2014 que apresentou 70,0\% de mulheres com faixa etária considerada fora de risco, mães de bebês com malformação. Em relação ao estado civil pode-se verificar que $25(71,4 \%)$ das mulheres eram solteiras, $9(25,7 \%)$ eram casadas e $1(2,9 \%)$ não tinha o campo de estado civil preenchido. A maioria das gestantes de alto risco eram solteiras, ou seja, convivem com a ausência do parceiro, o que, segundo Behrman, Kliegman e Jenson (2005), pode ser um fator associado à gestação de alto risco. Já Brito et al.,2010, em seu estudo afirma que mulheres que são mães tardiamente e que não possuem conjugue, possuem possibilidade de 11,4 vezes de terem um bebê com malformação, quando comparadas a mulheres que são mães entre 20 a 34 anos de idade.

O ramo de atividade materna mais prevalente foi de agricultora com 13 $(37,1 \%)$ mulheres. Quanto ao número de consultas pré-natais foi detectado que a maioria das mães fizeram mais de sete consultas, representando $60 \%$. O tipo de parto que mais ocorreu foi o parto cesáreo com 19 (54,3\%) partos do total corroborando com o estudo de Reis, Ferrari 2014 onde os partos de crianças com malformações também foram maioria com 52,2\%. Segundo Reis (2010), uma maior prevalência de parto cesáreo é motivo de preocupação, pois a prática indiscriminada desse tipo de parto não garante maiores benefícios ao $\mathrm{RN}$, já que o expõe a inúmeros riscos inerentes ao procedimento cirúrgico.

Os dados da Tabela 1 foram cruzados com os fatores de risco materno para malformações congênitas. Os resultados estatisticamente significativos obtidos foram os de malformações congênitas e estado civil materno $\left(x^{2=} 58,1\right.$ e $\left.p=0,04\right)$ que estão representados na Tabela 2. Não se observou evidência estatística de associação entre os tipos de malformações e os outros fatores de risco associados como idade materna, ocupação atual materna e número de consultas pré-natal. 
Tabela 2- Resultados do teste Qui-Quadrado entre os tipos de malformações e os fatores de risco associados.

\begin{tabular}{l|c|c}
\hline \multicolumn{1}{c|}{ Malformações Congênitas x Fatores de Risco } & $\mathbf{X}^{\mathbf{2}}$ & $\mathbf{P}$ \\
\hline Idade & 41,0 & 0,51 \\
\hline Estado Civil & 58,1 & 0,04 \\
\hline Ocupação Atual & 193,3 & 0,78 \\
\hline Número de Consultas pré-natal & 53,2 & 0,11 \\
\hline Tipo de parto & 21,7 & 0,41 \\
\hline
\end{tabular}

Fonte: PIMENTA, et al.,2019. Legenda: $x^{2}=$ resultado do teste qui-quadrado; $p=$ significância estatística do teste $(p<0,05)$. Cajazeiras, PB, Brasil.

Outro parâmetro avaliado no estudo foram os relacionados risco aos recémnascidos com malformação congênita encontrados na DN. Dentre os 35 RN com malformações $77,1 \%$ (27) são do sexo masculino e apenas $20 \%$ (7) do sexo feminino. Um estudo realizado em São Paulo por Mendes et. al. 2015, mostrou que os RN com malformações congênitas apresentaram uma maior frequência no sexo masculino $(50,9 \%)$, semelhante ao encontrado neste estudo. A raça predominante dos RN com malformação foi a raça parda com $91,4 \%$ dos casos e $77,1 \%$ apresentaram peso normal ao nascer.

Tabela 3 - Variáveis dos recém-nascidos com malformação congênita.

\begin{tabular}{ll|c|c}
\hline Sexo do recém-nascido & Feminino & $\mathbf{n}$ & $\mathbf{\%}$ \\
\hline & Masculino & 7 & 20 \\
\hline & Campo não preenchido & 27 & 77,1 \\
\hline Raça do Recém & & 1 & 2,9 \\
\hline & Branca & & \\
\hline & Parda & 3 & 8,6 \\
\hline Índice de Apgar no 5 & & 32 & 91,4 \\
\hline & Igual ou menor que 7 & & \\
\hline & Maior que 7 & 7 & 20 \\
\hline Peso do recém Nascido & Baixo peso $(<2,500 \mathrm{~kg})$ & 28 & 80 \\
\hline & Peso normal $(\geq 2,500 \mathrm{~kg}$ até $4,00 \mathrm{~kg}$ & 27 & 27,1 \\
\hline & Sobrepeso $(>4,000 \mathrm{~kg})$ & 1 & 2,9 \\
\hline
\end{tabular}

Fonte: PIMENTA et al., 2019. 


\section{CONCLUSÃO}

Foram constatados 35 casos de malformações congênitas entre os anos de 2010 e 2016 na cidade de Cajazeiras-PB, o que representa uma porcentagem de $0,27 \%$ do total de bebês que nasceram nesse período, essa baixa prevalência de malformações congênitas detectadas pode estar associada a subnotificação dos casos nas Declarações de Nascidos vivos. Dentre as malformações as mais frequentes foram as do sistema osteomuscular $(31,6 \%)$ com predominância da deformidade do pé. Não se observou relação estatísticas entre algumas variáveis materna em relação as malformações o que pode remeter que as MC detectadas neste estudo possam ser inferentes de fatores genéticos.

Sugere-se a inclusão na Declaração de nascidos vivos de campos com informações importantes como, se ocorreu doenças potencialmente causadoras de malformações durante a gestação, histórico familiar de malformações congênitas, uso de álcool, cigarro e medicamentos durante a gestação e histórico de doenças maternas durante a gestação. Tais informações podem ser de extrema valia na indicação de fatores de risco potenciais do desenvolvimento das malformações congênitas.

A quantidade significativa de Declarações de Nascido Vivo analisadas que não continham o campo de presença de malformação preenchido, assim como as Declarações de nascidos vivos que continham mais que não possuíam a descrição do tipo de malformação congênita deve ser ressaltado. Há uma necessidade de melhoria no preenchimento da DNV, o que pode ocorrer através de capacitação dos profissionais envolvidos no seu preenchimento, assim como profissionais de saúde treinados corretamente para atuarem na identificação e notificação das malformações congênitas na Maternidade em estudo. O preenchimento fiel e adequado deste documento é de extrema importância, visto que através dessas notificações pode-se diminuir ainda mais os números de $\mathrm{MC}$ com o rastreamento de fatores de risco associados. 


\section{REFERÊNCIAS BIBLIOGRÁFICAS}

ARRUDA, Tarciana Albuquerque Marenga de; AMORIM, Melania Maria Ramos de; SOUZA, Alex Sandro Rolland. Mortalidade determinada por anomalias congênitas em Pernambuco, Brasil, de 1993 A 2003. Revista da Associação Médica Brasileira, São Paulo, v. 54, n. 2, p. 122-126, 2008.

BEHRMAN, R. E.; KLIEGMAN, R. M.; JENSON, H. B. nelson: tratado de pediatria. 17. ed. Rio de Janeiro: Elsevier, 2005.

BRITO, Virgínia Rossana de Sousa et al. Malformações congênitas e fatores de risco materno em Campina Grande - Paraíba. Revista Rene, Fortaleza, v. 11, n. 2, p. 27-36, abr. 2010.

FONTOURA, Fabíola Chaves; CARDOSO, Maria Vera Lúcia Moreira Leitão. Association between congenital malformation and neonatal and maternal variables in neonatal units of a Northeast Brazilian city. Texto Contexto - Enfermagem. Florianópolis, v. 23, n. 4, p. 907-914, dez. 2014. FapUNIFESP http://dx.doi.org/10.1590/010407072014002320013.

MELO, WA; Zurita, RCM; Uchimura, TT; Marcon, SS. Anomalias congênitas: fatores associados à idade materna em município sul brasileiro, 200 a 2007. Revista Eletrônica d Enfermagem, vol. 12, n.1, p 73 a 82, 2010.

NHONCANSE, Geiza César; MELO, Débora Gusmão. Confiabilidade da Declaração de Nascido Vivo como fonte de informação sobre os defeitos congênitos no Município de São Carlos, São Paulo, Brasil. Ciência \& Saúde Coletiva, Rio de Janeiro, v. 17, n. 4, p. 955963, abr. 2012.

NUNES, Maria Diniz; TAVARES, Maria Clotilde Henriques. Perfil epidemiológico das malformações congênitas em recém-nascidos no estado do Tocantins no período de 2004 a 2008. 2010. 109 f. Dissertação (Mestrado) - Curso de Pós Graduação em Ciências da Saúde, Universidade de Brasília, Brasília, 2010.

Organização Mundial da Saúde. Classificação estatística internacional de doenças e problemas relacionados à saúde. São Paulo (SP): EDUSP; 2008.

PEREIRA, Danilo Alves Ponzi et al. Infecção congênita em pacientes matriculados em programa de referência materno infantil. Revista Paraense de Medicina, Umarizal, v. 29, n. 1, p. 31-38, jan. 2015.

PIMENTA, Marina de Souza; CALIL, Valdenise Martins Laurindo Tuma; KREBS, Vera Lúcia Jornada. Perfil das malformações congênitas no berçário anexo à maternidade do Hospital das Clínicas da Universidade de São Paulo. Revista de Medicina, São Paulo, v. 1, n. 89, p. 50-56, mar. 2010.

RAMOS, Aritana Pereira; OLIVEIRA, Maria Nice Dutra de; CARDOSO, Jefferson Paixão. Prevalência de malformações congênitas em recém-nascidos em hospital da rede pública. Revista Saúde.com, Bahia, v. 4, n. 1, p. 27-42, jan. 2008.

REIS, Lucimar de Lara Aire Silvestre dos; ALVES, Elioenai Dornelles. Perfil epidemiológico das malformações congênitas no município de Cáceres - Mato Grosso no período de 2004-2009. 2010. 98 f. Dissertação (Mestrado) - Curso de Pós Graduação em Ciências da Saúde, Universidade de Brasília, Brasília, 2010. 
REIS, Lucimar de Lara Aires Silvestre dos; FERRARI, Rogério. Malformações congênitas: perfil sociodemográfico das mães e condições de gestação. Revista de Enfermagem Ufpe On Line, Recife, v. 8, n. 1, p. 98-106, jan. 2014.

RODRIGUES, Lívia dos Santos et al. Características das crianças nascidas com malformações congênitas no município de São Luís, Maranhão, 2002-2011. Epidemiol. Serv. Saúde, Brasília, v. 2, n. 23, p.295-304, abr. 2014. 